\title{
Towards Understanding the Origin of Cosmic-Ray Positrons
}

M. Aguilar, ${ }^{26}$ L. Ali Cavasonza, ${ }^{1}$ G. Ambrosi, ${ }^{31}$ L. Arruda, ${ }^{24}$ N. Attig, ${ }^{21}$ P. Azzarello, ${ }^{15}$ A. Bachlechner, ${ }^{1}$ F. Barao, ${ }^{24}$ A. Barrau, ${ }^{16}$ L. Barrin, ${ }^{14}$ A. Bartoloni,${ }^{36}$ L. Basara, ${ }^{34}$ S. Başeğmez-du Pree,${ }^{17}$ R. Battiston,,${ }^{34,35,{ }^{*}}$ U. Becker,${ }^{10}$

M. Behlmann, ${ }^{10}$ B. Beischer, ${ }^{1}$ J. Berdugo, ${ }^{26}$ B. Bertucci, ${ }^{31,32}$ V. Bindi, ${ }^{19}$ W. de Boer, ${ }^{22}$ K. Bollweg, ${ }^{20}$ B. Borgia, ${ }^{36,37}$ M. J. Boschini, ${ }^{28}$ M. Bourquin, ${ }^{15}$ E. F. Bueno, ${ }^{38}$ J. Burger, ${ }^{10}$ W. J. Burger, ${ }^{34}$ X. D. Cai, ${ }^{10}$ M. Capell, ${ }^{10}$ S. Caroff, ${ }^{3}$ J. Casaus, ${ }^{26}$ G. Castellini, ${ }^{13}$ F. Cervelli, ${ }^{33}$ Y. H. Chang, ${ }^{43}$ G. M. Chen, ${ }^{6}$ H. S. Chen,${ }^{6,7}$ Y. Chen,${ }^{15}$ L. Cheng, ${ }^{39}$ H. Y. Chou, ${ }^{43}$ V. Choutko, ${ }^{10}$ C. H. Chung, ${ }^{1}$ C. Clark, ${ }^{20}$ G. Coignet,${ }^{3}$ C. Consolandi, ${ }^{19}$ A. Contin,,${ }^{8,9}$ C. Corti, ${ }^{19}$ M. Crispoltoni, ${ }^{31,32}$ Z. Cui, ${ }^{39}$ K. Dadzie ${ }^{10}$ Y. M. Dai, ${ }^{5}$ A. Datta, ${ }^{19}$ C. Delgado, ${ }^{26}$ S. Della Torre, ${ }^{28}$ M. B. Demirköz, ${ }^{2}$ L. Derome, ${ }^{16}$ S. Di Falco, ${ }^{33}$ F. Dimiccoli,,${ }^{34,14}$ C. Díaz, ${ }^{26}$ P. von Doetinchem, ${ }^{19}$ F. Dong,${ }^{30}$ F. Donnini, ${ }^{31, \dagger}$ M. Duranti, ${ }^{31}$ A. Egorov, ${ }^{10}$ A. Eline,${ }^{10}$ T. Eronen, ${ }^{44}$ J. Feng, ${ }^{10}$ E. Fiandrini, ${ }^{31,32}$ P. Fisher, ${ }^{10}$ V. Formato, ${ }^{31}$ Y. Galaktionov, ${ }^{10}$ R. J. García-López, ${ }^{23}$ C. Gargiulo, ${ }^{14}$ H. Gast, ${ }^{1}$ I. Gebauer, ${ }^{22}$ M. Gervasi, ${ }^{28,29}$ F. Giovacchini, ${ }^{26}$ D. M. Gómez-Coral, ${ }^{27}$ J. Gong,${ }^{30}$ C. Goy, ${ }^{3}$ V. Grabski, ${ }^{27}$ D. Grandi, ${ }^{28,29}$ M. Graziani, ${ }^{22}$ K. H. Guo, ${ }^{18}$ S. Haino, ${ }^{42}$ K. C. Han, ${ }^{25}$ Z. H. He ${ }^{18}$ M. Heil, ${ }^{10}$ T. H. Hsieh, ${ }^{10}$ H. Huang, ${ }^{42, \$}$ Z. C. Huang, ${ }^{18}$ M. Incagli, ${ }^{33}$ Yi Jia, ${ }^{10}$ H. Jinchi, ${ }^{25}$ K. Kanishev,${ }^{34}$ B. Khiali, ${ }^{31, \dagger}$ Th. Kirn, ${ }^{1}$ C. Konak, ${ }^{2}$ O. Kounina, ${ }^{10}$ A. Kounine, ${ }^{10}$ V. Koutsenko ${ }^{10}$ A. Kulemzin, ${ }^{10}$ G. La Vacca, ${ }^{28,29}$ E. Laudi, ${ }^{14}$ G. Laurenti, ${ }^{8}$ I. Lazzizzera, ${ }^{34,35}$ A. Lebedev, ${ }^{10}$ H. T. Lee, ${ }^{41}$ S. C. Lee, ${ }^{42}$ C. Leluc, ${ }^{15}$ J. Q. $\mathrm{Li},{ }^{30}$ Q. Li,${ }^{30}$ T. X. Li,${ }^{18}$ Z. H. Li, ${ }^{6}$ C. Light, ${ }^{19}$ C. H. Lin,${ }^{42}$ T. Lippert, ${ }^{21}$ F. Z. Liu, ${ }^{10}$ Hu Liu, ${ }^{10,43}$ Z. Liu, ${ }^{15}$ S. Q. Lu, ${ }^{42,}$ Y. S. Lu, ${ }^{6}$ K. Luebelsmeyer, ${ }^{1}$ F. Luo, ${ }^{39}$ J. Z. Luo, ${ }^{30}$ Xi Luo, ${ }^{19}$ S. S. Lyu, ${ }^{18}$ F. Machate, ${ }^{1}$ C. Mañá, ${ }^{26}$ J. Marín, ${ }^{26}$ T. Martin, ${ }^{20}$ G. Martínez, ${ }^{26}$ N. Masi, ${ }^{8}$ D. Maurin, ${ }^{16}$ A. Menchaca-Rocha, ${ }^{27}$ Q. Meng, ${ }^{30}$ D. C. Mo, ${ }^{18}$ M. Molero, ${ }^{26}$ P. Mott, ${ }^{20}$ L. Mussolin, ${ }^{31,32}$ T. Nelson, ${ }^{19}$ J. Q. Ni, ${ }^{18}$ N. Nikonov, ${ }^{1}$ F. Nozzoli, ${ }^{34}$ A. Oliva, ${ }^{26}$ M. Orcinha, ${ }^{24}$ M. Palermo, ${ }^{19}$ F. Palmonari, ${ }^{8,9}$ M. Paniccia ${ }^{15}$ A. Pashnin,${ }^{10}$ M. Pauluzzi, ${ }^{31,32}$ S. Pensotti, ${ }^{28,29}$ C. Perrina,${ }^{15}$ H. D. Phan,${ }^{10}$ N. Picot-Clemente, ${ }^{12}$

V. Plyaskin, ${ }^{10}$ M. Pohl,${ }^{15}$ V. Poireau, ${ }^{3}$ A. Popkow, ${ }^{19}$ L. Quadrani,${ }^{8,9}$ X. M. Qi,${ }^{18}$ X. Qin, ${ }^{10}$ Z. Y. Qu,${ }^{42, \|}$ P. G. Rancoita, ${ }^{28}$ D. Rapin, ${ }^{15}$ A. Reina Conde, ${ }^{23}$ S. Rosier-Lees, ${ }^{3}$ A. Rozhkov, ${ }^{10}$ D. Rozza, ${ }^{28,29}$ R. Sagdeev, ${ }^{11}$ C. Solano, ${ }^{10}$ S. Schael, ${ }^{1}$ S. M. Schmidt, ${ }^{21}$ A. Schulz von Dratzig, ${ }^{1}$ G. Schwering, ${ }^{1}$ E. S. Seo, ${ }^{12}$ B. S. Shan, ${ }^{4}$ J. Y. Shi, ${ }^{30}$ T. Siedenburg, ${ }^{1}$ J. W. Song,${ }^{39}$ Z. T. Sun, ${ }^{6}$ M. Tacconi,${ }^{28,29}$ X. W. Tang, ${ }^{6}$ Z. C. Tang, ${ }^{6}$ J. Tian, ${ }^{31,32}$ Samuel C. C. Ting, ${ }^{10,14}$ S. M. Ting, ${ }^{10}$ N. Tomassetti, ${ }^{31,32}$ J. Torsti ${ }^{44}$ T. Urban,${ }^{20}$ V. Vagelli, ${ }^{31,32}$ E. Valente, ${ }^{36,37}$ E. Valtonen, ${ }^{44}$ M. Vázquez Acosta, ${ }^{23}$ M. Vecchi,,${ }^{38,17}$ M. Velasco, ${ }^{26}$ J. P. Vialle, ${ }^{3}$ J. Vizán, ${ }^{26}$ L. Q. Wang, ${ }^{39}$ N. H. Wang, ${ }^{39}$ Q. L. Wang, ${ }^{5}$ X. Wang, ${ }^{10}$ X. Q. Wang, ${ }^{6,7}$ Z. X. Wang, ${ }^{18}$ J. Wei ${ }^{15}$ Z. L. Weng, ${ }^{10}$ H. Wu, ${ }^{30}$ R. Q. Xiong, ${ }^{30}$ W. Xu, ${ }^{10}$ Q. Yan, ${ }^{10}$ Y. Yang, ${ }^{40}$ H. Yi, ${ }^{30}$ Y. J. Yu, ${ }^{5}$ Z. Q. Yu, ${ }^{6}$ M. Zannoni, ${ }^{28,29}$ S. Zeissler, ${ }^{22}$ C. Zhang, ${ }^{6}$ F. Zhang, ${ }^{6}$ J. H. Zhang, ${ }^{30}$ Z. Zhang, ${ }^{10}$ F. Zhao, ${ }^{6,7}$ Z. M. Zheng, ${ }^{4}$ H. L. Zhuang, ${ }^{6}$ V. Zhukov, ${ }^{1}$ A. Zichichi, ${ }^{8,9}$ N. Zimmermann, ${ }^{1}$ and P. Zuccon ${ }^{34,35}$

(AMS Collaboration)

\author{
${ }^{1}$ I. Physics Institute and JARA-FAME, RWTH Aachen University, D-52056 Aachen, Germany \\ ${ }^{2}$ Department of Physics, Middle East Technical University (METU), 06800 Ankara, Turkey \\ ${ }^{3}$ Université Grenoble Alpes, Université Savoie Mont Blanc, CNRS, LAPP-IN2P3, F-74000 Annecy, France \\ ${ }^{4}$ Beihang University (BUAA), Beijing 100191, China \\ ${ }^{5}$ Institute of Electrical Engineering (IEE), Chinese Academy of Sciences, Beijing 100190, China \\ ${ }^{6}$ Institute of High Energy Physics (IHEP), Chinese Academy of Sciences, Beijing 100049, China \\ ${ }^{7}$ University of Chinese Academy of Sciences (UCAS), Beijing 100049, China \\ ${ }^{8}$ INFN Sezione di Bologna, I-40126 Bologna, Italy \\ ${ }^{9}$ Università di Bologna, I-40126 Bologna, Italy \\ ${ }^{10}$ Massachusetts Institute of Technology (MIT), Cambridge, Massachusetts 02139, USA \\ ${ }^{11}$ East-West Center for Space Science, University of Maryland, College Park, Maryland 20742, USA \\ ${ }^{12}$ IPST, University of Maryland, College Park, Maryland 20742, USA \\ ${ }^{13}$ CNR-IROE, I-50125 Firenze, Italy \\ ${ }^{14}$ European Organization for Nuclear Research (CERN), CH-1211 Geneva 23, Switzerland \\ ${ }^{15}$ DPNC, Université de Genève, CH-1211 Genève 4, Switzerland \\ ${ }^{16}$ Univ. Grenoble Alpes, CNRS, Grenoble INP, LPSC-IN2P3, F-38000 Grenoble, France \\ ${ }^{17}$ KVI-Center for Advanced Radiation Technology, University of Groningen, NL-9700 AB Groningen, the Netherlands \\ ${ }^{18}$ Sun Yat-Sen University (SYSU), Guangzhou 510275, China \\ ${ }^{19}$ Physics and Astronomy Department, University of Hawaii, Honolulu, Hawaii 96822, USA \\ ${ }^{20}$ National Aeronautics and Space Administration Johnson Space Center (JSC), \\ Jacobs Engineering, and Business Integra, Houston, Texas 77058, USA
}




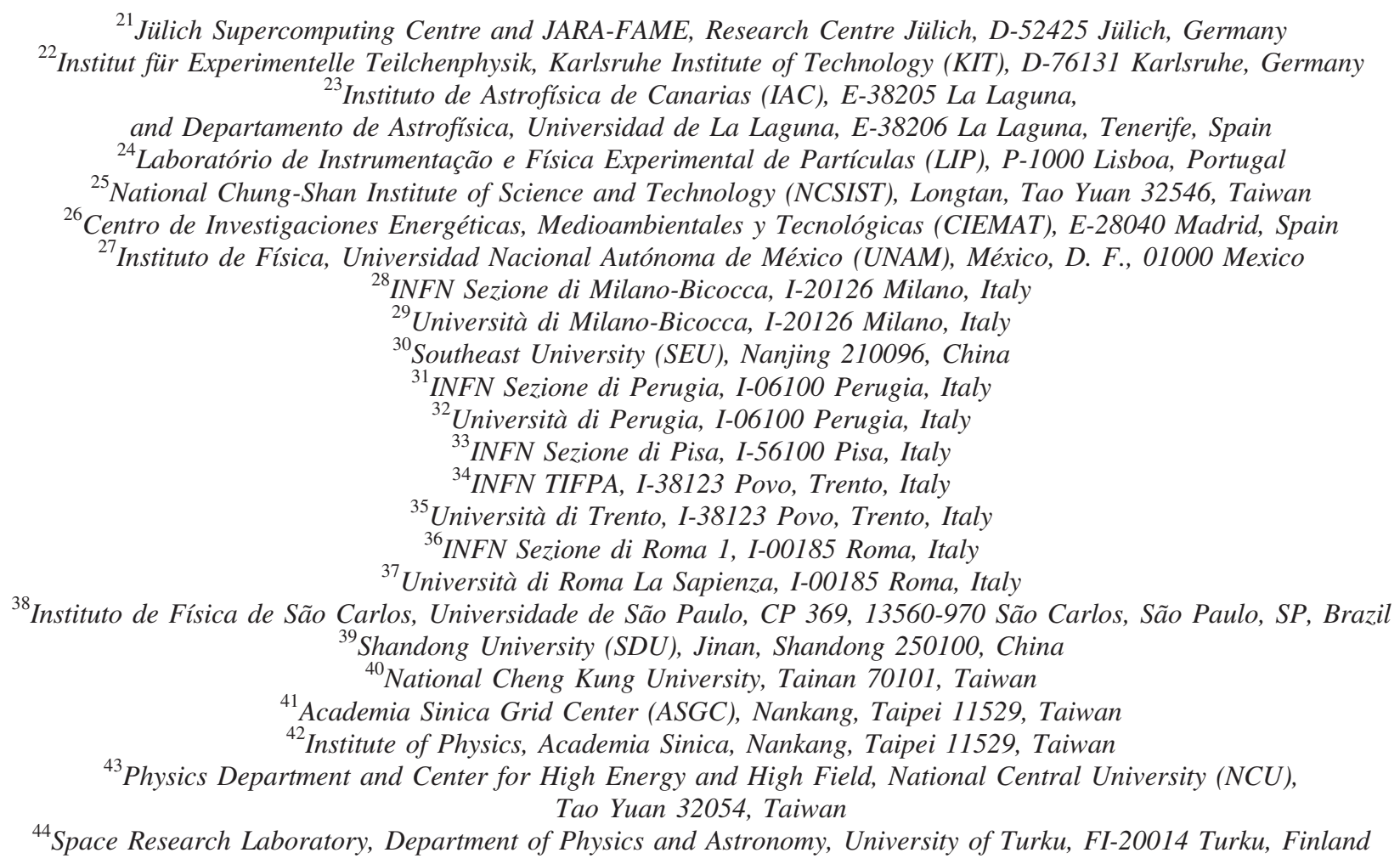

${ }^{21}$ Jülich Supercomputing Centre and JARA-FAME, Research Centre Jülich, D-52425 Jülich, Germany

${ }^{22}$ Institut für Experimentelle Teilchenphysik, Karlsruhe Institute of Technology (KIT), D-76131 Karlsruhe, Germany

${ }^{23}$ Instituto de Astrofísica de Canarias (IAC), E-38205 La Laguna,

and Departamento de Astrofísica, Universidad de La Laguna, E-38206 La Laguna, Tenerife, Spain

${ }^{24}$ Laboratório de Instrumentação e Física Experimental de Partículas (LIP), P-1000 Lisboa, Portugal

${ }^{25}$ National Chung-Shan Institute of Science and Technology (NCSIST), Longtan, Tao Yuan 32546, Taiwan

${ }^{26}$ Centro de Investigaciones Energéticas, Medioambientales y Tecnológicas (CIEMAT), E-28040 Madrid, Spain

${ }^{27}$ Instituto de Física, Universidad Nacional Autónoma de México (UNAM), México, D. F., 01000 Mexico

${ }^{28}$ INFN Sezione di Milano-Bicocca, I-20126 Milano, Italy

${ }^{29}$ Università di Milano-Bicocca, I-20126 Milano, Italy

${ }^{30}$ Southeast University (SEU), Nanjing 210096, China

${ }^{31}$ INFN Sezione di Perugia, I-06100 Perugia, Italy

${ }^{32}$ Università di Perugia, I-06100 Perugia, Italy

${ }^{33}$ INFN Sezione di Pisa, I-56100 Pisa, Italy

${ }^{34}$ INFN TIFPA, I-38123 Povo, Trento, Italy

${ }^{35}$ Università di Trento, I-38123 Povo, Trento, Italy

${ }^{36}$ INFN Sezione di Roma 1, I-00185 Roma, Italy

${ }^{37}$ Università di Roma La Sapienza, I-00185 Roma, Italy

${ }^{38}$ Instituto de Física de São Carlos, Universidade de São Paulo, CP 369, 13560-970 São Carlos, São Paulo, SP, Brazil

${ }^{39}$ Shandong University (SDU), Jinan, Shandong 250100, China

${ }^{40}$ National Cheng Kung University, Tainan 70101, Taiwan

${ }^{41}$ Academia Sinica Grid Center (ASGC), Nankang, Taipei 11529, Taiwan

${ }^{42}$ Institute of Physics, Academia Sinica, Nankang, Taipei 11529, Taiwan

${ }^{43}$ Physics Department and Center for High Energy and High Field, National Central University (NCU), Tao Yuan 32054, Taiwan

${ }^{44}$ Space Research Laboratory, Department of Physics and Astronomy, University of Turku, FI-20014 Turku, Finland

(Received 21 October 2018; revised manuscript received 4 December 2018; published 29 January 2019)

Precision measurements of cosmic ray positrons are presented up to $1 \mathrm{TeV}$ based on 1.9 million positrons collected by the Alpha Magnetic Spectrometer on the International Space Station. The positron flux exhibits complex energy dependence. Its distinctive properties are (a) a significant excess starting from $25.2 \pm 1.8 \mathrm{GeV}$ compared to the lower-energy, power-law trend, (b) a sharp dropoff above $284_{-64}^{+91} \mathrm{GeV}$, (c) in the entire energy range the positron flux is well described by the sum of a term associated with the positrons produced in the collision of cosmic rays, which dominates at low energies, and a new source term of positrons, which dominates at high energies, and (d) a finite energy cutoff of the source term of $E_{s}=810_{-180}^{+310} \mathrm{GeV}$ is established with a significance of more than $4 \sigma$. These experimental data on cosmic ray positrons show that, at high energies, they predominantly originate either from dark matter annihilation or from other astrophysical sources.

DOI: 10.1103/PhysRevLett.122.041102

The precision measurement of the positron flux in primary cosmic rays with the Alpha Magnetic Spectrometer (AMS) on the International Space Station (ISS) is presented with a particular emphasis on its behavior at high energies. The measurement is based on 1.9 million positrons collected by AMS from May 19, 2011 to November 12, 2017. This corresponds to a factor of three increase in statistics compared to our earlier results published four to five years ago $[1,2]$. Complementary to the

Published by the American Physical Society under the terms of the Creative Commons Attribution 4.0 International license. Further distribution of this work must maintain attribution to the author(s) and the published article's title, journal citation, and DOI. present data, we have also published the observation of low-energy $(<50 \mathrm{GeV})$ complex time structures in cosmic ray electron and positron fluxes during the same period as covered in this Letter [3]. Studies of light cosmic ray antimatter species, such as positrons, antiprotons, and antideuterons, are crucial for the understanding of new phenomena in the cosmos [4], because the yield of these particles from cosmic ray collisions is small. Our published data have generated widespread interest and discussions of the observed excess of high-energy positrons. The explanations of these results included three classes of models: annihilation of dark matter particles [5], acceleration of positrons to high energies in astrophysical objects [6], and production of high-energy positrons in the interactions of cosmic ray nuclei with interstellar gas [7]. Most of these explanations differ in their predictions for the behavior of 
cosmic ray positrons at high energies. The comprehensive paper on results for all elementary particles from the first 6.5 years of AMS operations, which includes details of the analysis and comparison with theoretical models, will be presented in a separate publication [8]. In this Letter, we present a precise measurement of primary cosmic ray positrons up to $1 \mathrm{TeV}$ and analyze the observation of changing behavior of the cosmic ray positron flux. These experimental results are crucial for understanding the origin of high-energy positrons in the cosmos.

Detector.- The description of the AMS detector is presented in Ref. [9]. The key detector elements used for the present analysis are the transition radiation detector (TRD) [10], the time of flight (TOF) counters [11], the silicon tracker [12], the permanent magnet [13], and the electromagnetic calorimeter (ECAL) [14]. AMS also has an array of 16 anticoincidence counters [15] and a ring imaging Čerenkov detector [16]. The detector performance on orbit is continuously monitored and it is steady over time.

The tracker accurately determines the particle trajectory and measures the rigidity $R$ (momentum divided by charge), the charge $|Z|$ in elementary charge units, and the charge sign of cosmic rays by multiple measurements of the coordinates in the magnetic field and the particle energy loss in the silicon layers. The tracker has nine layers, the first $L 1$ at the top of the detector, the second $L 2$ above the magnet, six $L 3$ to $L 8$ within the bore of the magnet, and the last $L 9$ above the ECAL. $L 2$ to $L 8$ constitute the inner tracker. For $|Z|=1$ particles the maximum detectable rigidity is $2 \mathrm{TV}$ over the 3-m lever arm and the charge resolution is $\Delta Z=0.05$. The TOF measures $|Z|$ with a resolution $\Delta Z=0.05$ and velocity $\beta$ with a resolution of $\Delta \beta / \beta^{2}=4 \%$. The TRD separates positrons $e^{+}$from protons $p$ using a $\Lambda_{\mathrm{TRD}}$ estimator constructed from the ratio of the log-likelihood probability of the $e^{ \pm}$hypothesis to that of the $p$ hypothesis in each layer [1]. The threedimensional imaging capability of the 17 radiation length ECAL allows for an accurate measurement of the $e^{ \pm}$ energy and of the shower shape. The $e^{ \pm}$energy, $E$, is calibrated at the top of AMS. An ECAL estimator $\Lambda_{\mathrm{ECAL}}$ [17] is used to differentiate $e^{ \pm}$from $p$ by exploiting their different shower shapes.

The entire detector has been extensively calibrated in a test beam at CERN with $e^{+}$and $e^{-}$from 10 to $290 \mathrm{GeV} / c$, with $p$ at 180 and $400 \mathrm{GeV} / c$, and with $\pi$ from 10 to $180 \mathrm{GeV} / c$, which produce transition radiation equivalent to $p$ up to $1.2 \mathrm{TeV} / c$. Measurements with 18 different energies and particles at 2000 positions were performed. A Monte Carlo program based on the GEANT4 10.1 package [18] is used to simulate physics processes and signals in the detector.

Event selection.-AMS has collected $1.07 \times 10^{11}$ cosmic rays in the first 6.5 years of operation. The data collection time used in this analysis includes only those seconds during which the detector was in normal operating conditions, AMS was pointing within $40^{\circ}$ of the local zenith, and the ISS was outside of the South Atlantic Anomaly. Because of the influence of the geomagnetic field, this collection time for galactic cosmic rays increases with rigidity reaching $1.51 \times 10^{8} \mathrm{~s}$ above $30 \mathrm{GeV}$.

Selected events are required to be downward going with $\beta>0.8$, to have a reconstructed shower in the ECAL with a matched track in the tracker and the TRD. For the analysis of the high-energy range, $E \geq 290 \mathrm{GeV}$, the track is required to pass through either $L 1$ or $L 9$. Track fitting quality criteria $\chi^{2} /$ d.o.f. $<20$ both in the bending and nonbending planes are applied to ensure good accuracy of the track reconstruction. The charge measurements in the TOF and the tracker are required to be consistent with $|Z|=1$.

The energy $E$ is required to be greater than 1.2 times the maximum Størmer cutoff [19] within the AMS field of view. An alternative procedure, with the cutoff calculated by backtracing particles from the top of AMS out to 50 Earth's radii [20] using the most recent International Geomagnetic Reference Field [21], yields the same results.

Events satisfying the selection criteria are classified into two categories-positive and negative rigidity data samples. In this Letter, we consider only the positive rigidity sample, which comprises positrons, background protons, and charge confusion electrons, that is, electrons which are reconstructed in the tracker with positive rigidity due to the finite tracker resolution or due to interactions with the detector materials.

The combination of information from the TRD, tracker, and ECAL enables the efficient separation of the positron signal events from these background sources using a template fitting technique. An energy dependent cut on $\Lambda_{\mathrm{ECAL}}$ is applied to remove the bulk of the proton background.

To distinguish positrons from charge confusion electrons, a charge confusion estimator $\Lambda_{\mathrm{CC}}^{e}$ is defined using the technique similar to that of Ref. [22], as presented in the Supplemental Material [23] including Fig. S1. The number of positrons and its statistical error in each bin are determined by fitting signal and background templates to data by varying their respective normalizations. The twodimensional $\left(\Lambda_{\mathrm{TRD}}-\Lambda_{\mathrm{CC}}^{e}\right)$ distribution is used to construct the templates. To fit the data, three templates are defined. The first two are for the positrons and protons reconstructed with correct charge sign; the last one is for the charge confusion electrons. The signal template is identical for positrons and for electrons if they are reconstructed with the correct charge sign, and therefore the positron signal template is taken from high purity electron data below $100 \mathrm{GeV}$ and from electron Monte Carlo simulation above $100 \mathrm{GeV}$. The charge confusion electron background template is from Monte Carlo simulation. The $e^{ \pm}$ Monte Carlo simulation is verified by the $e^{ \pm}$test beam data from 10 to $290 \mathrm{GeV}$, and by the AMS $e^{-}$data to $1 \mathrm{TeV}$. 
The proton background template is taken from high purity proton data.

An illustration of the fit to the data in the energy range [370-500] GeV is shown in Fig. S2 of the Supplemental Material [23]. The projections of the 2D data distribution onto the $\Lambda_{\mathrm{TRD}}$ and $\Lambda_{\mathrm{CC}}^{e}$ axes are shown together with the signal and background contributions.

In total, 1.9 million positrons are identified in the energy range from $0.5 \mathrm{GeV}$ to $1 \mathrm{TeV}$.

Data analysis.-The isotropic positron flux for the energy bin $E_{i}$ of width $\Delta E_{i}$ is given by

$$
\Phi_{e^{+}, i}=\frac{N_{i}}{A_{i}\left(1+\delta_{i}\right) T_{i} \Delta E_{i}},
$$

where the energy is defined at the top of AMS. The same energy binning as in our previous publications [2] is used. $N_{i}$ is the number of $e^{+}$in bin $i$ corrected for the small binto-bin migration using the unfolding procedure described in Ref. [24]. $A_{i}$ is the corresponding effective acceptance that includes geometric acceptance, and the trigger and selection efficiencies, and is calculated from Monte Carlo simulation. $T_{i}$ is the data collection time.

The small corrections $\delta_{i}$ are estimated by comparing the efficiencies in data and Monte Carlo simulation of every selection cut using information from the detectors unrelated to that cut. Because these efficiencies are the same for electrons and positrons, they are calculated from the high statistics electron samples. The corrections $\delta_{i}$ are found to have only a very small energy dependence from $-5 \%$ at $1 \mathrm{GeV}$, decreasing to $-2.4 \%$ at $10 \mathrm{GeV}$, and becoming constant at $-2.8 \%$ above $50 \mathrm{GeV}$.

The detailed study of the systematic errors is the key part of the analysis. Five sources of systematic error on the positron flux are identified.

The first source is related to the uncertainty in the template definitions, which includes the following two parts: the event selection and the statistical fluctuations. To examine the systematic errors associated with the selection, the selection cuts are varied such that the number of selected events in the corresponding template vary up to $30 \%$. Each variation yields three redefined templates. The variation of the number of positrons from the fits with the redefined templates is used to estimate the associated systematic error. This error amounts to $3 \%$ of the flux at $0.5 \mathrm{GeV},<0.5 \%$ between 1 and $500 \mathrm{GeV}$, and $7 \%$ for the energy bin [700-1000] GeV. The systematic error associated with fluctuations is measured by varying the shape of the templates within the statistical uncertainties. This error amounts to $<1 \%$ of the flux below $500 \mathrm{GeV}, 6 \%$ at [500-700] GeV, and $11 \%$ at [700-1000] GeV. These two errors are added in quadrature.

The second source is the uncertainty in the magnitude of the charge confusion. The amount of charge confusion is well reproduced by the Monte Carlo simulation. The corresponding systematic error accounts for the small differences between data and the Monte Carlo simulation. This error is negligible below $200 \mathrm{GeV}, 3 \%$ of the flux at [370-500] GeV, and $18 \%$ at [700-1000] GeV.

The third source is the uncertainty in the efficiency corrections $\delta_{i}$. The corresponding error amounts to $4 \%$ of the flux at $0.5 \mathrm{GeV}$, it decreases to $1.1 \%$ at $3 \mathrm{GeV}$, and slowly rises to $2.5 \%$ at $[700-1000] \mathrm{GeV}$. This includes a correlated systematic error on the flux normalization, which is estimated to be $1 \%$ of the flux independent of energy. This $1 \%$ error is subtracted in quadrature from the total systematic error for all the fits in this Letter.

The fourth source is the uncertainty in the magnitude of the event bin-to-bin migration due to the finite energy resolution. The bin widths $\Delta E_{i}$ are chosen to be at least two times the energy resolution to minimize migration effects [2]. Unfolding the measured fluxes shows that the bin-tobin migration is small: the corresponding error is $2 \%$ of the flux at $0.5 \mathrm{GeV}$ and decreases to $<0.2 \%$ above $10 \mathrm{GeV}$.

The fifth source is the uncertainty in the energy scale, which causes simultaneous shifts of the measured energies. As discussed in detail in Ref. [17], the energy scale error is $4 \%$ at $0.5 \mathrm{GeV}, 2 \%$ from 2 to $300 \mathrm{GeV}$, and $2.5 \%$ at $1 \mathrm{TeV}$.

The total systematic error of the positron flux is taken as the quadratic sum of the four sources: definition of templates, charge confusion, the efficiency corrections, and bin-to-bin migration. The energy scale error is treated as an uncertainty of the bin boundaries.

Several independent analyses were performed on the same data sample by different study groups [8]. The results of those analyses are consistent with the results presented in this Letter.

Results.-The measured positron flux including statistical and systematic errors is presented in Table SI of the Supplemental Material [23] as a function of the energy at the top of AMS. The table also includes $\tilde{E}$ values, calculated for a flux $\propto E^{-3}$ [25] and the corresponding energy scale errors. The precision of our data allows an accurate study of the properties of cosmic positrons without using the traditional positron fraction, defined as the ratio $\Phi_{e^{+}} /\left(\Phi_{e^{+}}+\Phi_{e^{-}}\right)$, which includes the energy dependence of the electrons.

The presented results are consistent with our earlier data $[1,2]$, which were based on $1 / 3$ of the current statistics. The current results greatly improve the accuracy and energy reach of our measurement. For instance, the error on the flux in the previous highest energy bin [370-500] GeV [2] is reduced from $30 \%$ to $12 \%$ and the energy reach is increased by a factor of 2 from $500 \mathrm{GeV}$ to $1 \mathrm{TeV}$.

Figure 1 shows the measured positron spectrum (i.e., the flux scaled by $\tilde{E}^{3}, \tilde{E}^{3} \Phi_{e^{+}}$). In this and the subsequent figures, the error bars correspond to the quadratic sum of statistical and systematic errors. As seen, at low energies from 0.5 to $7.10 \mathrm{GeV}$ there is a significant time variation of the spectrum due to solar modulation effects [indicated by a red band [3] ]. Starting from $7.10 \mathrm{GeV}$ this variation 


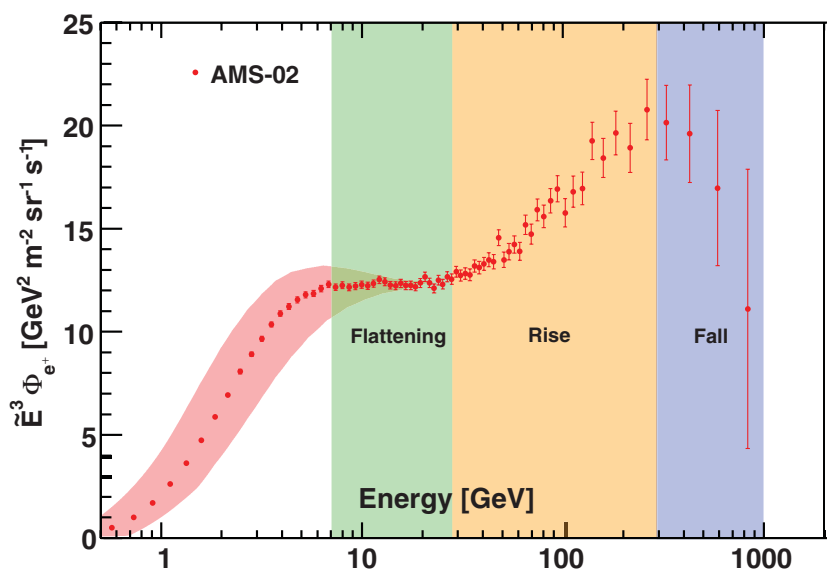

FIG. 1. For display purposes, the positron flux, $\Phi_{e^{+}}$is traditionally presented scaled by $\tilde{E}^{3}$. The resulting AMS positron spectrum, $\tilde{E}^{3} \Phi_{e^{+}}$, (red data points) is shown as a function of energy $\tilde{E}$. The time variation of the flux at low energies due to solar modulation [3] is indicated by the red band. To guide the eye, the vertical color bands indicate the energy ranges corresponding to changing behavior of the spectrum: flattening, rising, and falling spectrum (see text).

gradually vanishes and the average positron spectrum is flattening from 7.10 to $27.25 \mathrm{GeV}$ (green vertical band). At higher energies, where solar modulation effects are small [3], it exhibits a complex structure: a rise from 27.25 to $290 \mathrm{GeV}$ (orange vertical band), a maximum at $\sim 290 \mathrm{GeV}$ followed by a sharp fall.

Figure 2 shows the AMS result together with earlier experiments [26-31]. The AMS data significantly extend the measurements into the uncharted high-energy region.

To examine the energy dependence of the positron flux in a model independent way, the flux spectral index $\gamma$ is calculated from the equation

$$
\gamma=d\left[\log \left(\Phi_{e^{+}}\right)\right] / d[\log (E)],
$$

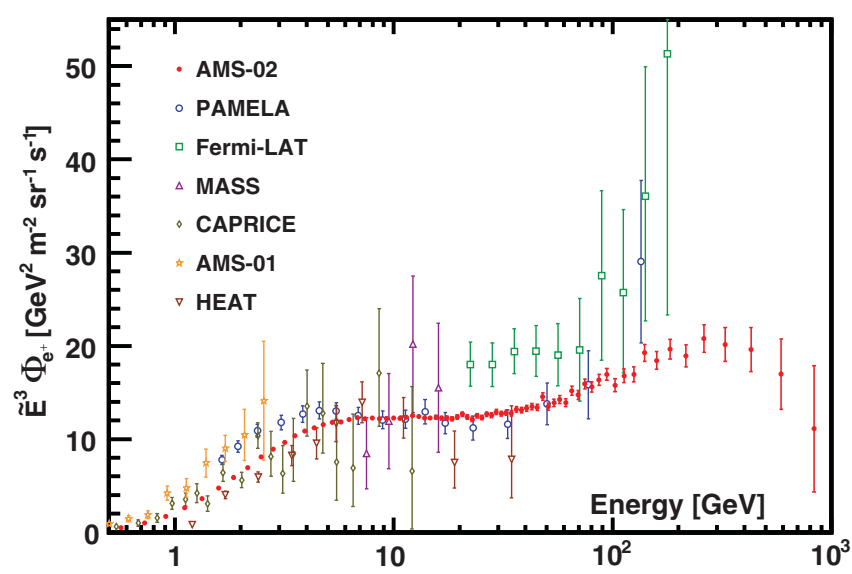

FIG. 2. The AMS positron spectrum $\left(\tilde{E}^{3} \Phi_{e^{+}}\right)$together with earlier measurements from PAMELA [26], Fermi-LAT [27], MASS [28], CAPRICE [29], AMS-01 [30], and HEAT [31]. over nonoverlapping energy intervals which are chosen to have sufficient sensitivity to the spectral index. The energy interval boundaries are 3.36, 5.00, 7.10, 10.32, 17.98, $27.25,55.58,90.19,148.81,290$, and $1000 \mathrm{GeV}$ that combine several energy bins defined in Table SI of the Supplemental Material [23]. The results are presented in Fig. 3(a). They are stable against the variation of energy interval boundaries as verified by shifting the boundaries to higher and lower values by one or two energy bins [see Fig. S3 of the Supplemental Material [23]]. As seen in Fig. 3(a), the positron spectral index exhibits complex behavior. It decreases (softens) rapidly with energy below $\sim 7 \mathrm{GeV}$. In the energy range [7.10-27.25] GeV, it is nearly energy independent, with an average $\gamma=-2.99 \pm 0.01$. It then rises (hardens) to an average $\gamma=-2.72 \pm 0.04$ in the energy range [55.58-148.81] GeV. Above $148.81 \mathrm{GeV}$ the spectral index experiences significant decrease reaching $\gamma=-3.35 \pm 0.32$ in the highest energy interval [290-1000] GeV.

To determine the transition energy $E_{0}$ where the spectral index starts rising, we use a double power-law approximation

$\Phi_{e^{+}}(E)= \begin{cases}C(E / 55.58 \mathrm{GeV})^{\gamma}, & E \leq E_{0} ; \\ C(E / 55.58 \mathrm{GeV})^{\gamma}\left(E / E_{0}\right)^{\Delta \gamma}, & E>E_{0} .\end{cases}$

A fit to data is performed in the energy range [7.1055.58] GeV. The results are presented in Fig. 3(b). The fit yields $E_{0}=25.2 \pm 1.8 \mathrm{GeV}$ for the energy where the spectral index increases and $\chi^{2} /$ d.o.f. $=23 / 31$. The significance of this increase is established at more than $6 \sigma$, as illustrated in Fig. S4 of the Supplemental Material [23]. The energy $E_{0}$ corresponds to the start of a significant excess of the positron flux compared to the lower-energy trends. Note that the choice of the constant $55.58 \mathrm{GeV}$, corresponding to the fit range boundary, defines only the flux normalization $C$. It does not affect fitted values of $\gamma$ and $\Delta \gamma$.

To determine the transition energy where the spectral index starts decreasing, we use Eq. (3) to fit the data in the energy range [55.58-1000] GeV. The results are presented in Fig. 3(c). The fit yields $E_{0}=284_{-64}^{+91} \mathrm{GeV}$ for the energy of the spectral energy decrease and $\chi^{2} /$ d.o.f. $=13 / 16$. The significance of the spectral index decrease at $284_{-64}^{+91} \mathrm{GeV}$ is established at more than $3 \sigma$, as illustrated in Fig. S5 of the Supplemental Material [23].

This complex behavior of the positron flux (as illustrated in Fig. 3) is consistent with the existence of a new source of high-energy positrons with a characteristic cutoff energy, whether of dark matter [5] or other astrophysical [6] origin. It is not consistent with the exclusive secondary production of positrons in collisions of cosmic rays [32].

The accuracy of the AMS data allows for a detailed study of the properties of the new source of positrons up to $1 \mathrm{TeV}$. In this Letter, we present the analysis of the positron 

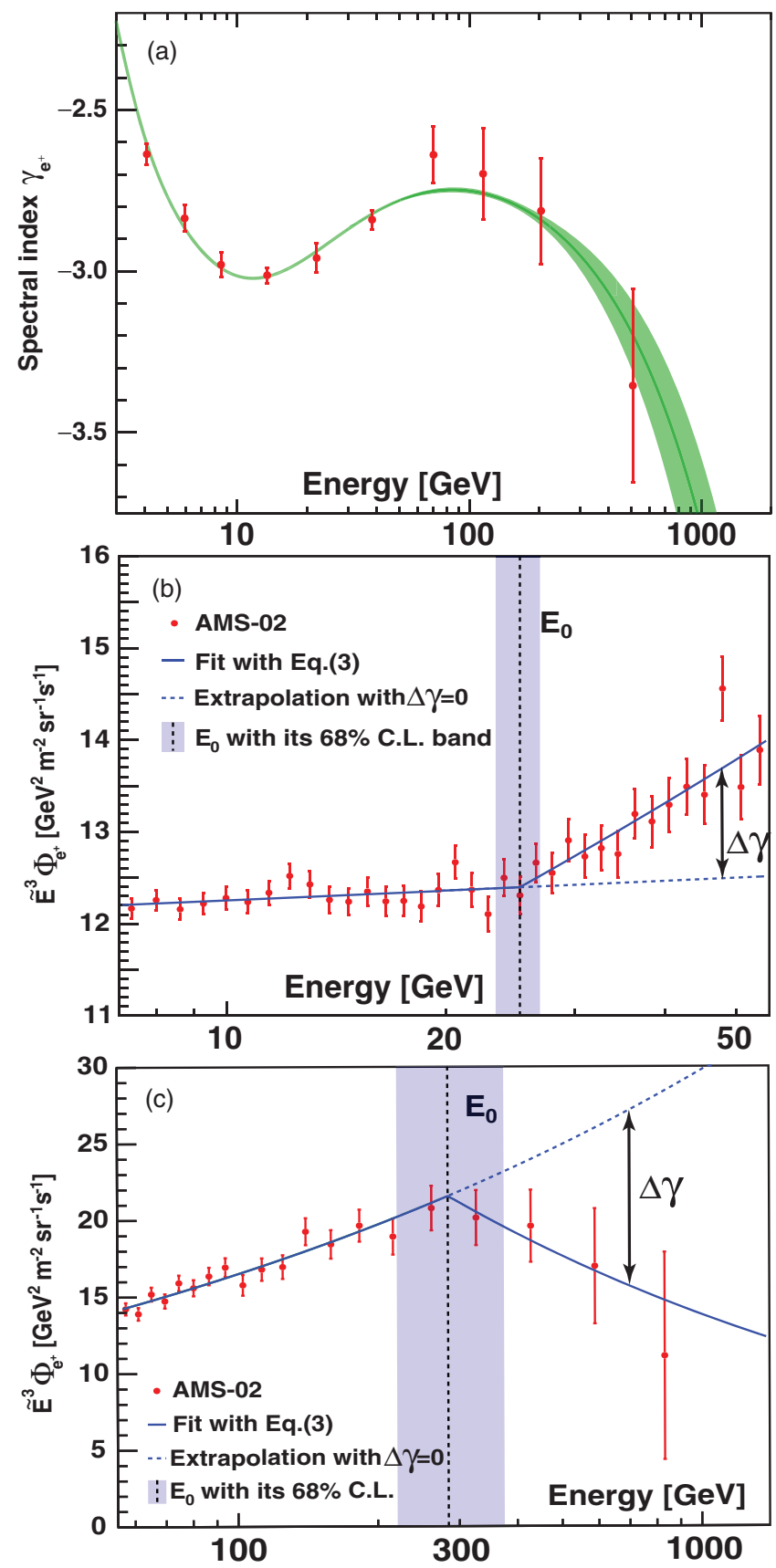

FIG. 3. (a) The spectral index of the AMS positron flux in nonoverlapping energy intervals (red data points). The spectral index has complex energy dependence with a significant decrease towards higher energies. (b),(c) A double power-law fit of Eq. (3) to the flux in the energy ranges [7.10-55.58] and [55.58-1000] GeV, respectively. The red data points are the measured positron flux values scaled by $\tilde{E}^{3}$. The fitted functions are represented by the blue lines. The vertical dashed lines and the bands correspond to the value and the error of the energies $E_{0}$ where the changes of the spectral index occur. The dashed blue lines are the extrapolations of the power law below $E_{0}$ into the higher-energy regions. $\Delta \gamma$ is the magnitude of the spectral index change, see Eq. (3). The green band in (a) represents the $68 \%$ C.L. interval of the minimal model fit of Eq. (4) to the positron flux (see text). flux using a minimal model $[1,33]$. In this model, $\Phi_{e^{+}}$is parametrized as the sum of a diffuse term and a source term

$$
\begin{aligned}
\Phi_{e^{+}}(E)= & \frac{E^{2}}{\hat{E}^{2}}\left[C_{d}\left(\hat{E} / E_{1}\right)^{\gamma_{d}}\right. \\
& \left.+C_{s}\left(\hat{E} / E_{2}\right)^{\gamma_{s}} \exp \left(-\hat{E} / E_{s}\right)\right] .
\end{aligned}
$$

The diffuse term describes the low-energy part of the flux dominated by the positrons produced in the collisions of ordinary cosmic rays with the interstellar gas. It is characterized by a normalization factor $C_{d}$ and a spectral index $\gamma_{d}$. The source term has an exponential cutoff, which describes the high-energy part of the flux dominated by a source. It is characterized by a normalization factor $C_{s}$, a spectral index $\gamma_{s}$, and a cutoff energy $E_{s}$. In order to account for solar modulation effects, the force-field approximation [34] is used, with the energy of particles in the interstellar space $\hat{E}=E+\varphi_{e^{+}}$, where the effective solar potential $\varphi_{e^{+}}$ accounts for the solar effects.

Explicitly, we have chosen the first term of Eq. (4) based on the general trend of the commonly used cosmic ray propagation models $[32,35,36]$, even though all have large uncertainties, but all show a maximum of the spectrum below $10 \mathrm{GeV}$. The second term is based on our data in Figs. 3(b) and 3(c), which show that the spectrum changes its characteristics at $25.2 \mathrm{GeV}$, where the spectrum shows the distinct increase, and at $284 \mathrm{GeV}$, where the spectrum shows a sharp dropoff.

The constant $E_{1}$ is chosen to be $7.0 \mathrm{GeV}$ to minimize the correlation between parameters $C_{d}$ and $\gamma_{d}$, and the constant $E_{2}$ is chosen to be $60.0 \mathrm{GeV}$ to minimize correlation between the parameters $C_{s}$ and $\gamma_{s}$. The fit of Eq. (4) to the measured flux yields the inverse cutoff energy $1 / E_{s}=$ $1.23 \pm 0.34 \mathrm{TeV}^{-1}$ corresponding to $E_{s}=810_{-180}^{+310} \mathrm{GeV}$ and $\chi^{2} /$ d.o.f. $=50 / 68$. A complete list of the fit parameters is given in Table SII of the Supplemental Material [23]. All the source term parameters, $C_{s}, \gamma_{s}$, and $1 / E_{s}$, are found to be time independent whereas the solar modulation parameter $\varphi_{e^{+}}$and the diffuse flux parameters $C_{d}$ and $\gamma_{d}$ exhibit some time dependence, as presented in Fig. S6 of the Supplemental Material [23].

The result of the fit is presented in Fig. 4. As expected, the diffuse term dominates at low energies and then gradually vanishes with increasing energy. The source term dominates the positron spectrum at high energies. It is the contribution of the source term that leads to the observed excess of the positron flux above $25.2 \pm 1.8 \mathrm{GeV}$. The dropoff of the flux above $284_{-64}^{+91} \mathrm{GeV}$ is very well described by the sharp exponential cutoff of the source term. Note that the choice of the $E_{1}$ and $E_{2}$ constants does not affect the shapes nor the magnitudes of the diffuse and the source terms shown in Fig. 4. The energy dependence of the spectral index corresponding to the results of the fit of Eq. (4) is shown in Fig. 3(a). 


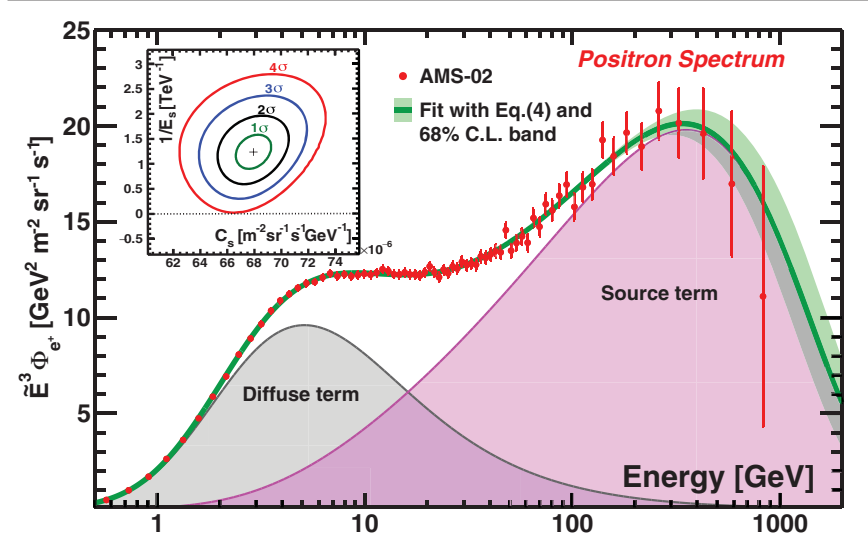

FIG. 4. The fit of Eq. (4) (green line) to the positron flux in the energy range [0.5-1000] GeV together with the $68 \%$ C.L. interval (green band). The exponential cutoff of the source term is determined to be $810_{-180}^{+310} \mathrm{GeV}$ from the fit. The red data points represent the measured positron flux values scaled by $\tilde{E}^{3}$. The source term contribution is represented by the magenta area and the diffuse term contribution by the gray area. The insert shows projections of the regions of $1 \sigma$ (green contour), $2 \sigma$ (black contour), $3 \sigma$ (blue contour), and $4 \sigma$ (red contour) significance of the $1 / E_{s}$ measurement onto the plane of parameters $1 / E_{s}-C_{s}$ (see text).

To study the significance of the $1 / E_{s}$ measurement we varied all six fit parameters to find the regions in sixdimensional (6D) parameter space corresponding to the confidence levels from 1 to $5 \sigma$ with a step of $0.01 \sigma$. As an example, the insert in Fig. 4 shows projections of the $6 \mathrm{D}$ regions of $1 \sigma$ (green line, $68.26 \%$ C.L.), $2 \sigma$ (black line, 95.54\% C.L.), $3 \sigma$ (blue line, $99.74 \%$ C.L.), and $4 \sigma$ (red line, $99.99 \%$ C.L.) onto the plane of parameters $\left(1 / E_{s}-C_{s}\right)$. Detailed analysis shows that a point where the parameter $1 / E_{s}$ reaches 0 corresponds to a confidence level of $4.07 \sigma$; i.e., the significance of the source term energy cutoff is established at more than $4 \sigma$, or that the positron flux in the entire energy range cannot be described by a sum of two power-law functions at the $99.99 \%$ C.L.

An analysis of the arrival directions of electrons and positrons was presented in Ref. [1]. A similar analysis was performed using the positron data of this Letter [37]. The positron flux is found to be consistent with isotropy; the upper limit on the amplitude of the dipole anisotropy is $\delta<0.019$ at the $95 \%$ C.L. for energies above $16 \mathrm{GeV}$.

In conclusion, we have presented the precision measurements of the positron flux from $0.5 \mathrm{GeV}$ to $1 \mathrm{TeV}$, with a detailed study of systematic errors based on 1.9 million positrons. The positron flux shows complex energy dependence. Its distinctive properties are (a) a significant excess starting from $25.2 \pm 1.8 \mathrm{GeV}$ compared to the lower-energy trends, (b) a sharp dropoff above $284_{-64}^{+91} \mathrm{GeV}$, (c) in the entire energy range the positron flux is well described by the sum of a diffuse term associated with the positrons produced in the collision of cosmic rays, which dominates at low energies, and a new source term of positrons, which dominates at high energies, and (d) a finite energy cutoff of the source term of $E_{s}=810_{-180}^{+310} \mathrm{GeV}$ is established with a significance of more than $4 \sigma$. These experimental data on cosmic ray positrons show that, at high energies, they predominantly originate either from dark matter annihilation or from other astrophysical sources.

We thank former NASA Administrator Daniel S. Goldin for his dedication to the legacy of the ISS as a scientific laboratory and his decision for NASA to fly AMS as a DOE payload. We also acknowledge the continuous support of the NASA leadership, particularly William $\mathrm{H}$. Gerstenmaier, and of the JSC and MSFC flight control teams that have allowed AMS to operate optimally on the ISS for over seven years. We are grateful for the support of Jim Siegrist and his staff of the DOE including resources from the National Energy Research Scientific Computing Center under Contract No. DE-AC02-05CH11231. We also acknowledge the continuous support from MIT and its School of Science, Michael Sipser, and Boleslaw Wyslouch. Research supported by São Paulo Research Foundation (FAPESP) Grant No. 2014/19149-7, Brazil; CAS, NSFC, MOST, the provincial governments of Shandong, Jiangsu, Guangdong, and the China Scholarship Council, China; CNRS/IN2P3, CNES, Enigmass, and the ANR, France; Pascale Ehrenfreund, DLR under Grant No. 50001403 and JARA-HPC under Project No. JARA0052, Germany; INFN and ASI under ASI-INFN Agreements No. 2013-002-R.0 and No. 2014037-R.0, Italy; the Consejo Nacional de Ciencia y Tecnología and UNAM, Mexico; FCT under Grant No. PTDC/FIS/122567/2010, Portugal; CIEMAT, IAC, CDTI, and SEIDI-MINECO under Grants No. ESP201787055-C2-1-P, No. SEV-2015-0548, No. MDM-20150509, and No. RyC-2013-14660, Spain; the Swiss National Science Foundation (SNSF), federal and cantonal authorities, Switzerland; Academia Sinica and the Ministry of Science and Technology (MOST) under Grants No. 1032112-M-006-018-MY3, No. 105-2112-M-001-003, and No. CDA-105-M06, former Presidents of Academia Sinica Yuan-Tseh Lee and Chi-Huey Wong and former Ministers of MOST Maw-Kuen Wu and Luo-Chuan Lee, Taiwan; the Turkish Atomic Energy Authority under Grant No. 2017TAEK(CERN)A5.H6.F2-15, Turkey; and NSF Grants No. 14255202 and No. 1551980, Wyle Laboratories Grant No. 2014/T72497, and NASA NESSF Grant No. HELIO15F-0005, the United States of America. We gratefully acknowledge the strong support from CERN including Fabiola Gianotti, and the CERN IT department including Bernd Panzer-Steindel, and from the European Space Agency including Johann-Dietrich Wörner and Simonetta Di Pippo. We are grateful for important physics discussions with Pasquale Blasi, Fiorenza Donato, Jonathan Ellis, Jonathan Feng, Mischa Malkov, Igor Moskalenko, Subir Sarkar, and Steven Weinberg. 
*Also at ASI, I-00133 Roma, Italy.

${ }^{\dagger}$ Also at ASI Space Science Data Center (SSDC), I-00133 Roma, Italy.

${ }^{\ddagger}$ Also at Wuhan University, Wuhan 430072, China.

${ }^{\S}$ Also at Sun Yat-Sen University (SYSU), Guangzhou 510275, China.

"Also at Nankai University, Tianjin 300071, China.

[1] M. Aguilar et al., Phys. Rev. Lett. 110, 141102 (2013); L. Accardo et al., Phys. Rev. Lett. 113, 121101 (2014).

[2] M. Aguilar et al., Phys. Rev. Lett. 113, 121102 (2014); 113, 221102 (2014).

[3] M. Aguilar et al., Phys. Rev. Lett. 121, 051102 (2018).

[4] F. Donato, N. Fornengo, and P. Salati, Phys. Rev. D 62, 043003 (2000); M. Cirelli, R. Franceschini, and A. Strumia, Nucl. Phys. B800, 204 (2008); P. Blasi, Phys. Rev. Lett. 103, 051104 (2009); I. Cholis and D. Hooper, Phys. Rev. D 88, 023013 (2013); K. Blum, K. Chun Yu Ng, R. Sato, and M. Takimoto, Phys. Rev. D 96, 103021 (2017).

[5] M. S. Turner and F. Wilczek, Phys. Rev. D 42, 1001 (1990); J. Ellis, AIP Conf. Proc. 516, 21 (2000); H. C. Cheng, J. L. Feng, and K. T. Matchev, Phys. Rev. Lett. 89, 211301 (2002); G. Kane, R. Lu, and S. Watson, Phys. Lett. B 681, 151 (2009); J. Kopp, Phys. Rev. D 88, 076013 (2013); C. H. Chen, C. W. Chiang, and T. Nomura, Phys. Lett. B 747, 495 (2015); H. C. Cheng, W. C. Huang, X. Huang, I. Low, Y. L. Sming Tsia, and Q. Yuan, J. Cosmol. Astropart. Phys. 03 (2017) 041; Y. Bai, J. Berger, and S. Lu, Phys. Rev. D 97, 115012 (2018).

[6] P. D. Serpico, Astropart. Phys. 39-40, 2 (2012); T. Linden and S. Profumo, Astrophys. J. 772, 18 (2013); P. Mertsch and S. Sarkar, Phys. Rev. D 90, 061301 (2014); N. Tomassetti and F. Donato, Astrophys. J. Lett. 803, L15 (2015); D. Hooper, I. Cholis, T. Linden, and K. Fang, Phys. Rev. D 96, 103013 (2017); W. Liu, X. J. Bi, S. J. Lin, B. B. Wang, and P. F. Yin, Phys. Rev. D 96, 023006 (2017); M. Kachelrieß, A. Neronov, and D. V. Semikoz, Phys. Rev. D 97, 063011 (2018); S. Profumo, J. Reynoso-Cordova, N. Kaaz, and M. Silverman, Phys. Rev. D 97, 123008 (2018).

[7] P. Lipari, Phys. Rev. D 95, 063009 (2017); R. Cowsik, B. Burch, and T. Madziwa-Nussinov, Astrophys. J. 786, 124 (2014); K. Blum, B. Katz, and E. Waxman, Phys. Rev. Lett. 111, 211101 (2013).

[8] AMS Collaboration, Phys. Rev. D (to be published).

[9] A. Kounine, Int. J. Mod. Phys. E 21, 1230005 (2012); S. Rosier-Lees, in Proceedings of the 19th Symposium on Astroparticle Physics in the Netherlands, Beekbergen, 2014 (unpublished); S. C. C. Ting, Nucl. Phys. B243-244, 12 (2013); S. C. Lee, in Proceedings of the 20th International Conference on Supersymmetry and Unification of Fundamental Interactions (SUSY 2012), Beijing, 2012 (unpublished); M. Aguilar, in Proceedings of the XL International Meeting on Fundamental Physics, Centro de Ciencias de Benasque Pedro Pascual, 2012 (unpublished); S. Schael, in Proceedings of the 10th Symposium on Sources and Detection of Dark Matter and Dark Energy in the Universe, Los Angeles, 2012 (unpublished); B. Bertucci, Proc. Sci. EPS-HEP (2011) 67; M. Incagli, AIP Conf. Proc. 1223, 43 (2010); R. Battiston, Nucl. Instrum. Methods Phys. Res., Sect. A 588, 227 (2008).
[10] F. Hauler et al., IEEE Trans. Nucl. Sci. 51, 1365 (2004); Ph. Doetinchem et al., Nucl. Instrum. Methods Phys. Res., Sect. A 558, 526 (2006); Th. Kirn, Nucl. Instrum. Methods Phys. Res., Sect. A 706, 43 (2013).

[11] V. Bindi et al., Nucl. Instrum. Methods Phys. Res., Sect. A 743, 22 (2014) and references therein.

[12] B. Alpat et al., Nucl. Instrum. Methods Phys. Res., Sect. A 613, 207 (2010).

[13] K. Lübelsmeyer et al., Nucl. Instrum. Methods Phys. Res., Sect. A 654, 639 (2011).

[14] C. Adloff et al., Nucl. Instrum. Methods Phys. Res., Sect. A 714, 147 (2013).

[15] Ph. von Doetinchem, W. Karpinski, Th. Kirn, K. Lübelsmeyer, St. Schael, and M. Wlochal, Nucl. Phys. B197, 15 (2009).

[16] M. Aguilar-Benitez et al., Nucl. Instrum. Methods Phys. Res., Sect. A 614, 237 (2010); F. Giovacchini, Nucl. Instrum. Methods Phys. Res., Sect. A 766, 57 (2014).

[17] A. Kounine, Z. Weng, W. Xu, and C. Zhang, Nucl. Instrum. Methods Phys. Res., Sect. A 869, 110 (2017).

[18] S. Agostinelli et al., Nucl. Instrum. Methods Phys. Res., Sect. A 506, 250 (2003); J. Allison et al., IEEE Trans. Nucl. Sci. 53, 270 (2006).

[19] C. Størmer, The Polar Aurora (Oxford University Press, London, 1950).

[20] J. Alcaraz et al., Phys. Lett. B 484, 10 (2000).

[21] C. C. Finlay et al., Geophys. J. Int. 183, 1216 (2010); E. Thébault et al., Earth Planets Space 67, 79 (2015).

[22] M. Aguilar et al., Phys. Rev. Lett. 117, 091103 (2016).

[23] See Supplemental Material at http://link.aps.org/ supplemental/10.1103/PhysRevLett.122.041102 for a description of the charge confusion analysis; the tabulated positron flux and its statistical and systematic errors; and figures related to event selection, the stability of the spectral index, the significance of the fits of Eq. (3), and the time stability of the fit of Eq. (4).

[24] M. Aguilar et al., Phys. Rev. Lett. 114, 171103 (2015).

[25] G. D. Lafferty and T. R. Wyatt, Nucl. Instrum. Methods Phys. Res., Sect. A 355, 541 (1995). We have used Eq. (6) with $\tilde{E} \equiv x_{l w}$.

[26] O. Adriani et al., Phys. Rev. Lett. 111, 081102 (2013).

[27] M. Ackermann et al., Phys. Rev. Lett. 108, 011103 (2012).

[28] C. Grimani et al., Astron. Astrophys. 392, 287 (2002).

[29] M. Boezio et al., Adv. Space Res. 27, 669 (2001).

[30] M. Aguilar et al., Phys. Lett. B 646, 145 (2007).

[31] S. W. Barwick et al., Astrophys. J. 498, 779 (1998); M. A. DuVernois et al., Astrophys. J. 559, 296 (2001).

[32] I. V. Moskalenko and A. W. Strong, Astrophys. J. 493, 694 (1998); A. E. Vladimirov, S. W. Digel, G. Jóhannesson, P. F. Michelson, I. V. Moskalenko, P. L. Nolan, E. Orlando, T. A. Porter, and A. W. Strong, Comput. Phys. Commun. 182, 1156 (2011).

[33] L. Ali Cavasonza, H. Gast, M. Kramer, M. Pellen, and S. Schael, Astrophys. J. 839, 36 (2017). This Letter also uses a minimal model analysis assuming a charge symmetric source term, and the AMS positron, electron, and combined fluxes based on 2.5 year of data [2]. 
[34] R. A. Caballero-Lopez and H. Moraal, J. Geophys. Res. 109, A01101 (2004); L. Gleeson and W. Axford, Astrophys. J. 154, 1011 (1968).

[35] R. Trotta, G. Jóhannesson, I. V. Moskalenko, T. A. Porter, R. Ruiz de Austri, and A. W. Strong, Astrophys. J. 729, 106 (2011).
[36] T. Delahaye, J. Lavalle, R. Lineros, F. Donato, and N. Fornengo, Astron. Astrophys. 524, A51 (2010).

[37] M. A. Velasco, Ph. D. thesis, Universidad Complutense de Madrid, 2018; S. Zeissler, Ph. D. thesis, Karlsruhe Institute of Technology, 2018. 